\title{
Kinetic Alfvén solitary and rogue waves in superthermal plasmas
}

\author{
A. S. Bains, Bo Li** and Li-Dong Xia \\ Shandong Provincial Key Laboratory of Optical \\ Astronomy \&f Solar-Terrestrial Environment, \\ School of Space Science and Physics, \\ Shandong University at Weihai, 264209, Weihai, PR China
}

(Dated: June 2, 2021)

\begin{abstract}
We investigate the small but finite amplitude solitary Kinetic Alfvén waves (KAWs) in low $\beta$ plasmas with superthermal electrons modeled by a kappa-type distribution. A nonlinear Korteweg-de Vries $(\mathrm{KdV})$ equation describing the evolution of KAWs is derived by using the standard reductive perturbation method. Examining the dependence of the nonlinear and dispersion coefficients of the $\mathrm{KdV}$ equation on the superthermal parameter $\kappa$, plasma $\beta$ and obliqueness of propagation, we show that these parameters may change substantially the shape and size of solitary KAW pulses. Only sub-Alfvénic, compressive solitons are supported. We then extend the study to examine kinetic Alfvén rogue waves by deriving a nonlinear Schrödinger equation from the KdV equation. Rational solutions that form rogue wave envelopes are obtained. We examine how the behavior of rogue waves depends on the plasma parameters in question, finding that the rogue envelopes are lowered with increasing electron superthermality whereas the opposite is true when the plasma $\beta$ increases. The findings of this study may find applications to low $\beta$ plasmas in astrophysical environments where particles are superthermally distributed.
\end{abstract}

\footnotetext{
*Electronic address: bbl@sdu.edu.cn
} 


\section{INTRODUCTION}

Kinetic Alfvén waves (KAWs) play an important role in transporting and dissipating energies in a large number of space and heliospheric plasma environments [1, 2]. Unlike the shear Alfvén waves in ideal Magnetohydrodynamics (MHD), KAWs are dispersive, and have an electrostatic field parallel to the external magnetic field. This distinct behavior takes place when kinetic effects associated with the finite ion Larmor radius and/or finite inertial length have to be taken into account. For instance, when the perpendicular wavelength is comparable to the ion gyroradius, ions no longer follow the magnetic lines of force, whereas electrons still do due to their small Larmor radii. This produces a charge separation, and the corresponding coupling of shear Alfvén waves to electrostatic modes results in KAWs as a normal mode of the system.

Dispersion combined with nonlinearity lead to the formation of solitary KAWs. In 1976, Hasegawa and Mima [7] were the first to find the exact solitary KAW solutions using kinetic theory for a small but finite plasma beta such that $m_{e} / m_{i} \ll \beta \ll 1$, where $m_{e} / m_{i}$ denotes the electron to ion mass ratio. These authors neglected ion inertia in the ion continuity equation and also the ion contribution to the parallel current density in Ampere's law, and derived the energy balance equation governing the solitary wave solutions of density humps. Incorporating these two effects while addressing the same situation where $m_{e} / m_{i} \ll \beta \ll 1$, Yu and Shukla [8] showed that an upper limit exists for the amplitude of exact solitary Alfvén

waves. Shukla et al.[9] again extended the work of Hasegawa and Mima [7] by considering the electron inertia to be the dominant contributor to wave dispersion as happens when $\beta \ll$ $m_{e} / m_{i} \ll 1$. They found super-Alfvénic solitons with density depression and also derived the KdV equation from the Sagdeev potential equation. Examining the same model as Shukla et al. [9] but capitalizing on the concept of ion drift velocity, Kalita and Kalita [10] investigated the existence of exact nonlinear KAWs and showed that both super- and sub- Alfvénic rarefactive solitons exist, depending on the angle between the direction of propagation and the external magnetic field. Das et al. [11] studied the stability of the solitary KAWs considered by Shukla et al. [9]. After deriving the modified KdV equation and then carrying out a stability analysis using the small- $k$ perturbation expansion method of Rowlands and Infeld [12, 13], these authors found that the growth rate of the instability decreases with increasing angle between the direction of propagation and the external uniform magnetic 
field. Ghosh and Das [14] again studied the stability of solitary KAWs for a plasma with low but finite $\beta$ values by considering the Boltzmann-distributed electrons. It was observed that the growth rate of the instability attains a maximum for perturbations given along a direction lying in the plane containing the external magnetic field and the propagation direction of solitary waves. Over the last two decades or so these investigations attracted the attention of many researchers to study solitary kinetic Alfvén waves in a variety of plasma models [15 21]. Some investigations were also focused on the effects of dust particles on solitary KAWs [2224]. We note that in most of the above-mentioned theoretical investigations the particle distributions are assumed to be a Maxwellian.

There are many circumstances in which the well-known Maxwellian distribution is not a proper description of the plasma species [25 27]. On theoretical grounds, [28] showed that a plasma in the presence of superthermal particles is subject to velocity-space diffusion, which will lead to a power law distribution at speeds much higher than the electron thermal speed. Observationally speaking, the $\kappa$ distribution [29] has been suggested to be more appropriate for describing most space plasmas than a Maxwellian. The presence of a high-energy tail component in a kappa distribution considerably changes the rate of resonant energy transfer between particles and plasma waves, so the conditions for various plasma instabilities may differ substantially for the two distributions. While there are many studies on the linear and nonlinear characteristics of electrostatic waves using these types of distributions [30 34], only a few exist that examine solitary KAWs in the presence of non-thermally distributed electrons [35 38]. Bandyopadhyay and Das [35] studied the stability of KAWs and ionacoustic waves in nonthermal plasmas using the Rowlands-Infeld method. Solitary KAWs of arbitrary amplitude for systems where electrons are nonthermally distributed is studied by Roychoudhury [36]. An exact Sagdeev potential equation was derived and it was found that both hump and dip solitons exist depending upon the nonthermal parameters for electrons. Recently, Gogoi and Khan [37] studied the arbitrary amplitude kinetic Alfvén solitons using warm adiabatic ions and kappa-distributed electrons in a magnetized plasma. The system was shown to support compressive and double layer solutions, and it was demonstrated that the spectral index $\kappa$ has significant effects on the shape and size of the solitary KAWs.

Recently rogue waves have become of considerable interest in the broad areas of nonlinear fibre optics [39], optical systems [40, 41], atmospheric research [42] and plasmonics [43]. Rogue waves are short-lived phenomena appearing suddenly out of normal waves. Excess 
amount of energy accumulated in a small region makes the oscillations much stronger than the surrounding waves, with the initial process forming rogue waves usually attributed to the modulational instability. In the context of plasma physics, rogue waves have been studied for both electrostatic [44 46] and electromagnetic [47-49] modes. Shukla and Moslem [47] studied the formation of left- and right-hand circularly polarized Alfvénic rogue waves due to the nonlinear interaction between circularly polarized dispersive Alfvén waves and lowfrequency electrostatic perturbations. The amplitude of Alfvénic rogue waves decreases with increasing plasma number density, and increases with increasing magnetic field strength. Panwar et al.[48] studied the Alfvén rogue and solitary waves in an MHD plasma. It was shown that compressional solitons exist, and the amplitude of the solitons increases with increasing soliton speed and decreases with the plasma $\beta$. In addition, the envelope of the compressional Alfvén rogue waves decreases with increasing $\beta$, while electron inertia supports compressional Alfvén rogue waves with larger amplitudes. Very recently, El-Awady et al.[49] examined magnetosonic rogue waves in a two-component plasma, and found that a stronger magnetic field leads to a smaller rouge wave amplitude. These investigations stimulate our interest in studying rogue wave solutions in the context of KAWs.

The aim of the present manuscript is to elucidate the effects of electron superthermality, as manifested through the commonly observed kappa distribution, on the propagation characteristics of nonlinear kinetic Alfvén solitons. An exact KdV equation for the solitary KAWs is derived with the reductive perturbation method for the first time. The results agree closely with what was found with the well-known Sagdeev potential approach. A nonlinear Schrödinger wave equation is then derived to study kinetic Alfvén rogue waves.

The organization of this manuscript is as follows. Section II presents the detailed derivation of the KdV equation, while section III examines the rogue wave solutions. The last section summarizes the present study.

\section{MODEL EQUATIONS AND DERIVATION OF THE KDV EQUATION}

We consider a magnetized electron-ion plasma with small but finite $\beta$ such that $m_{e} / m_{i} \ll$ $\beta \ll 1$. Ions are described by a set of fluid moment equations, while electrons are modeled by a kappa velocity distribution. We take the ambient magnetic field $\mathbf{B}_{0}$ to be in the $z$-direction. 
The quasi-neutrality condition is used, i.e., $n_{e}=n_{i}=n$. The two-potential theory [50] is employed to handle the perpendicular $\left(\phi_{\perp}\right)$ and parallel $\left(\phi_{\|}\right)$potentials, justifiable for low- $\beta$ plasmas. We assume that the propagation occurs in the $x-z$ plane. The model equations can be written in normalized form as [8]

$$
\begin{array}{r}
\frac{\partial n}{\partial t}+\frac{\partial\left(n v_{x}\right)}{\partial x}+\frac{\partial\left(n v_{z}\right)}{\partial z}=0 \\
\frac{\partial v_{z}}{\partial t}+v_{x} \frac{\partial v_{z}}{\partial x}+v_{z} \frac{\partial v_{z}}{\partial z}=-\frac{\beta}{2} \frac{\partial \phi_{\|}}{\partial z}, \\
v_{x}=-\frac{\beta}{2} \frac{\partial^{2} \phi_{\perp}}{\partial x \partial t}, \\
\frac{\partial^{4}\left(\phi_{\perp}-\phi_{\|}\right)}{\partial x^{2} \partial z^{2}}=\frac{2}{\beta}\left[\frac{\partial^{2} n}{\partial t^{2}}+\frac{\partial\left(n v_{z}\right)}{\partial t \partial z}\right],
\end{array}
$$

with the electron density given by [31, 32$]$

$$
n=\left(1-\frac{\phi_{\|}}{\kappa-3 / 2}\right)^{-\kappa+1 / 2}
$$

Here time is normalized by the reciprocal of the ion cyclotron frequency $\Omega_{c i}$, and the spatial coordinates by the ion inertial length $d_{i}=c / \omega_{p i}$ where $c$ is the light speed and $\omega_{p i}$ is the ion plasma frequency. Besides, $n$ is normalized by the ambient density $n_{0}$, the velocity components $\left(v_{x}, v_{z}\right)$ by the Alfvén speed $v_{A}=c B_{0} /\left(n_{0} m_{i} / \epsilon_{0}\right)^{1 / 2}$, and the potentials $\left(\phi_{\perp}, \phi_{\|}\right)$ by $T / e$. Here $T$ is a constant electron temperature, and $e$ is the absolute electron charge. To derive the $\mathrm{KdV}$ equation we use the following stretching coordinates

$$
\xi=\epsilon^{1 / 2}\left(l_{x} x+l_{z} z-M t\right), \quad \tau=\epsilon^{3 / 2} t
$$

where $\epsilon$ is a small dimensionless parameter representing the strength of nonlinearity, $l_{x}$ and $l_{z}$ are the directional cosines in the $x$ - and $z$-directions, respectively $\left(l_{x}^{2}+l_{z}^{2}=1\right)$. When appropriate, we will also denote the propagation obliqueness with $\theta=\arccos l_{z}$. Besides, $M$ is the wave phase speed in units of $v_{A}$. The dependent variables can be expanded as (see [14, 35])

$$
\begin{aligned}
& n=1+\epsilon n^{(1)}+\epsilon^{2} n^{(2)}+\ldots \ldots \\
& v_{z}=\epsilon v_{z}^{(1)}+\epsilon^{2} v_{z}^{(2)}+\ldots \ldots \\
& v_{x}=\epsilon v_{x}^{(1)}+\epsilon^{2} v_{x}^{(2)}+\ldots \ldots \\
& \phi_{\|}=\epsilon \phi_{\|}^{(1)}+\epsilon^{2} \phi_{\|}^{(2)}+\ldots \ldots \\
& \phi_{\perp}=\phi_{\perp}^{(1)}+\epsilon \phi_{\perp}^{(2)}+\ldots .
\end{aligned}
$$


We now plug these expansions into the basic equations, and compare terms at different orders of $\epsilon$. At $\epsilon^{3 / 2}$ and $\epsilon^{2}$ one finds

$$
\begin{aligned}
& -c_{1} M \phi_{\|}^{(1)}+l_{x} v_{x}^{(1)}+l_{z} v_{z}^{(1)}=0, \\
& M v_{z}^{(1)}=\frac{l_{z} \beta}{2} \phi_{\|}^{(1)}, \\
& v_{x}^{(1)}=\frac{l_{x} \beta M}{2} \frac{\partial^{2} \phi_{\perp}^{(1)}}{\partial \xi^{2}}, \\
& l_{x}^{2} l_{z}^{2} \frac{\partial^{2} \phi_{\perp}^{(1)}}{\partial \xi^{2}}=\frac{2}{\beta}\left(c_{1} M^{2} \phi_{\|}^{(1)}-l_{z} M v_{z}^{(1)}\right),
\end{aligned}
$$

where $c_{1}=(\kappa-1 / 2) /(\kappa-3 / 2)$. Solving Eqs.(8) to (11) yields the following dispersion relation,

$$
2 c_{1} M^{4}-\left(\beta+2 c_{1}\right) l_{z}^{2} M^{2}+\beta l_{z}^{4}=0,
$$

which gives rise to two different modes. One is a kinetic Alfvén mode corresponding to

$$
M^{2}=l_{z}^{2},
$$

the other corresponds to the well-studied ion acoustic mode [e.g., 51, 52] and is not to be pursued here. At $\epsilon^{3 / 2}$, one finds

$$
\begin{aligned}
& c_{1} \frac{\partial \phi_{\|}^{(1)}}{\partial \tau}-c_{1} M \frac{\partial \phi_{\|}^{(2)}}{\partial \xi}-c_{2} M \frac{\partial\left(\phi_{\|}^{(1)}\right)^{2}}{\partial \xi}+l_{x} \frac{\partial v_{x}^{(2)}}{\partial \xi}+c_{1} l_{x} \frac{\partial\left(\phi_{\|}^{(1)} v_{x}^{(1)}\right)}{\partial \xi}+l_{z} \frac{\partial v_{z}^{(2)}}{\partial \xi}+c_{1} l_{z} \frac{\partial\left(\phi_{\|}^{(1)} v_{z}^{(1)}\right)}{\partial \xi}=0 \\
& v_{x}^{(2)}=\frac{l_{x} \beta M}{2} \frac{\partial^{2} \phi_{\perp}^{(2)}}{\partial \xi^{2}}-\frac{l_{x} \beta}{2} \frac{\partial \phi_{\perp}^{(1)}}{\partial \xi \partial \tau} \\
& \frac{\partial v_{z}^{(1)}}{\partial \tau}-M \frac{\partial v_{z}^{(2)}}{\partial \xi}+l_{x} v_{x}^{(1)} \frac{\partial v_{z}^{(1)}}{\partial \xi}+l_{z} v_{z}^{(1)} \frac{\partial v_{z}^{(1)}}{\partial \xi}+\frac{l_{z} \beta}{2} \frac{\partial \phi_{\|}^{(2)}}{\partial \xi}=0
\end{aligned}
$$

From Eq.(4) at $\epsilon^{3}$ one finds

$$
\begin{aligned}
l_{x}^{2} l_{z}^{2} \frac{\partial^{4}\left(\phi_{\perp}^{(2)}-\phi_{\|}^{(1)}\right)}{\partial \xi^{4}} & =\frac{2}{\beta}\left[c_{1} M^{2} \frac{\partial^{2} \phi_{\|}^{(2)}}{\partial \xi^{2}}+c_{2} M^{2} \frac{\partial^{2}\left(\phi_{\|}^{(1)}\right)^{2}}{\partial \xi^{2}}-2 c_{1} M \frac{\partial^{2} \phi_{\|}^{(1)}}{\partial \tau \partial \xi}\right. \\
& \left.+l_{z} \frac{\partial v_{z}^{(1)}}{\partial \xi \partial \tau}-c_{1} l_{z} M \frac{\partial^{2}\left(\phi_{\|}^{(1)} v_{z}^{(1)}\right)}{\partial \xi^{2}}-l_{z} M \frac{\partial^{2} v_{z}^{(2)}}{\partial \xi^{2}}\right]
\end{aligned}
$$


where $c_{2}=\left(c_{1} / 2\right)(\kappa+1 / 2)(\kappa-3 / 2)$. With the aid of Eqs. (8) to (11), the above equations simplify to

$$
\begin{aligned}
& a_{2} \frac{\partial \phi_{\|}^{(1)}}{\partial \tau}-c_{1} M \frac{\partial \phi_{\|}^{(2)}}{\partial \xi}+a_{3} \phi_{\|}^{(1)} \frac{\partial \phi_{\|}^{(1)}}{\partial \xi}+l_{z} \frac{\partial v_{z}^{(2)}}{\partial \xi}+\frac{l_{x}^{2} \beta M}{2} \frac{\partial^{3} \phi_{\perp}^{(2)}}{\partial \xi^{3}}=0 \\
& \frac{l_{z} \beta}{2 M} \frac{\partial \phi_{\|}^{(1)}}{\partial \tau}-M \frac{\partial v_{z}^{(2)}}{\partial \xi}+a_{4} \phi_{\|}^{(1)} \frac{\partial \phi_{\|}^{(1)}}{\partial \xi}+\frac{l_{z} \beta}{2} \frac{\partial \phi_{\|}^{(2)}}{\partial \xi}=0 \\
& l_{x}^{2} \frac{\partial^{3}\left(\phi_{\perp}^{(2)}-\phi_{\|}^{(1)}\right)}{\partial \xi^{3}}=\frac{2 c_{1} M^{2}}{\beta l_{z}^{2}} \frac{\partial \phi_{\|}^{(2)}}{\partial \xi}+a_{5} \phi_{\|}^{(1)} \frac{\partial \phi_{\|}^{(1)}}{\partial \xi}+a_{6} \frac{\partial \phi_{\|}^{(1)}}{\partial \tau}-\frac{2 M}{l_{z} \beta} \frac{\partial v_{z}^{(2)}}{\partial \xi}
\end{aligned}
$$

where

$$
\begin{aligned}
& a_{1}=\frac{2}{l_{x}^{2} \beta}-\frac{l_{z}^{2}}{l_{x}^{2} M^{2}}, \quad a_{2}=1-\frac{a_{1} l_{x}^{2} \beta}{2}, \quad a_{3}=a_{1} l_{x}^{2} \beta M-M+l_{z}^{2} \beta / M, \\
& a_{4}=\frac{a_{1} l_{x}^{2} l_{z} \beta^{2}}{4}+\frac{l_{z}^{3} \beta^{2}}{4 M^{2}}, \quad a_{5}=\frac{1}{l_{z}^{2}}\left(\frac{4 c_{2} M^{2}}{\beta}-2 c_{1} l_{z}^{2}\right), \quad a_{6}=\frac{1}{l_{z}^{2}}\left(\frac{l_{z}^{2}}{M}-\frac{4 c_{1} M}{\beta}\right) .
\end{aligned}
$$

Eliminating second order quantities from Eqs.(18) to (20) with the help of Eq.(12), one arrives at the following $\mathrm{KdV}$ equation

$$
\frac{\partial \psi}{\partial \tau}+A \psi \frac{\partial \psi}{\partial \xi}+B \frac{\partial^{3} \psi}{\partial \xi^{3}}=0,
$$

where $\psi=\phi_{\|}^{(1)}$. We note that the parallel potential $\phi_{\|}$in dimensionless form is required to be small for the reductive perturbation approach to be applicable. However, $\phi_{\|}^{(1)}$ is allowed to be of order unity, since it appears immediately after the smallness parameter in the expansion (17). Furthermore, the nonlinearity and dispersion coefficients, $A$ and $B$, are given by

$$
\begin{aligned}
& A=-c_{1} l_{z}, \\
& B=-\frac{\beta l_{x}^{2} l_{z}}{4\left(c_{1}-\beta / 2\right)} .
\end{aligned}
$$

The steady state solution to the KdV equation can be obtained by transforming $\xi$ and $\tau$ to $\eta=\xi+m_{0} \tau$ and $\tau=\tau$, where $m_{0}$ is a constant velocity normalized by $v_{A}$, and by imposing appropriate boundary conditions for localized perturbations, namely $\psi \rightarrow 0, \partial \psi / \partial \eta \rightarrow 0$, $\partial^{2} \psi / \partial \eta^{2} \rightarrow 0$ when $\eta \rightarrow \pm \infty$. A possible solution is given by

$$
\psi=\psi_{m} \operatorname{sech}^{2}(\eta / \Delta)
$$

where the soliton amplitude and width, $\psi_{m}$ and $\Delta$, are given by

$$
\psi_{m}=3 m_{0} / A, \text { and } \Delta=\sqrt{4 B / m_{0}},
$$


respectively.

The characteristics of the solitary KAWs as described by Eq.(25) can be examined as follows. First, let us note that with $\beta \ll 1$, both $A$ and $B$ are negative given that $c_{1}>1$. For $\Delta$ to be meaningful, $m_{0}$ has to be negative. Furthermore, if letting $\eta=\epsilon^{1 / 2} \tilde{\eta}$, then one notices from Eq.([6) that $\tilde{\eta}=l_{x} x+l_{z}\left[z-\left(1+\epsilon m_{0} / l_{z}\right) t\right]$. A negative $m_{0}$ therefore renders the parallel propagation speed in dimensionless form, $1+\epsilon m_{0} / l_{z}$, smaller than unity, meaning that only sub-Alfvénic, compressive $\left(\psi_{m} \propto m_{0} / A>0\right)$ solitons are permitted. This agrees with the original Hasegawa-Mima discussion [7] (hereafter HM) even though a Maxwellian was assumed for the electron distribution therein. If rewriting $1+\epsilon m_{0} / l_{z}$ as $1 / K_{z}$ as defined in HM, then for weakly nonlinear waves $\delta_{M}=\left(K_{z}^{2}-1\right) \ll 1$, one finds that $m_{0} \approx-\delta_{M} l_{z} /(2 \epsilon)$. The density perturbation $\tilde{n} \equiv n-1 \approx \epsilon n^{(1)}=\epsilon c_{1} \psi^{(1)}$ may then be expressed as

$$
\tilde{n}=\tilde{n}_{M} \operatorname{sech}^{2}(\tilde{\eta} / \tilde{\Delta})
$$

where

$$
\tilde{n}_{M}=\frac{3 c_{1}}{2} \delta_{M}, \quad \tilde{\Delta}=\sqrt{\frac{3 \beta}{1-\beta /\left(2 c_{1}\right)} \frac{l_{x}^{2}}{\tilde{n}_{M}}},
$$

which generalizes the original solution (Eq.(12) in HM) to the case where electrons are $\kappa$ distributed. In the limiting case $\kappa \rightarrow \infty$, one finds that $c_{1}=1$ and Eq.(12) in HM is readily recovered.

As is well-known, the deviation from the Maxwellian behavior of the electron background is measured by the value of spectral index $\kappa$. So the effects of the electron superthermality on the soliton amplitude $\left(\psi_{m}\right)$ and width $(\Delta)$ are investigated in Fig, 1, which plots the full soliton profile $(\psi)$ as a function of $\eta$ for fixed values of $\beta=0.05$ and $\theta=40^{\circ}$. For illustrative purpose, a value of -0.3 is adopted for $m_{0}$. It can be seen that with increasing $\kappa$ value (i.e., decreasing superthermality) both the amplitude and width of solitary pulses increase, a feature readily understandable given that $c_{1}$ decreases monotonically with increasing $\kappa$. Moreover, while the amplitude does not vary significantly with varying $\kappa$ for $\kappa \gtrsim 10$, at smaller values of $\kappa$ it is a sensitive function of $\kappa$ with the sensitivity being particularly pronounced when $\kappa$ approaches $3 / 2$. This means that stronger deviations from a Maxwellian support weaker and narrower solitons.

Moving on to Fig, 2, we note that the plasma $\beta$ does not affect the amplitude of solitary waves but it has significant effects on their widths, which tend to increase with increasing $\beta$. 
Both features are readily understandable with Eq.(26) is approximately proportional to $\sqrt{\beta}$ for small enough $\beta$. One then sees that plasma systems with high $\beta$ values tend to support wide solitary KAWs.

The effect of propagation angle $\theta$ with respect to the external magnetic field is examined in Fig 3 , where the full soliton profile is plotted as a function of $\eta$. The plasma $\beta$ and $\kappa$ are fixed at 0.05 and 2, respectively. From Fig 3 it can be seen that both the pulse amplitude and width increase with increasing obliqueness, which once again can be understood with Eq.(26) . We note that the width $\Delta \propto \sqrt{l_{x}^{2} l_{z}}=\sqrt{\sin ^{2} \theta \cos \theta}$ is zero when $\theta$ is either $0^{\circ}$ or $90^{\circ}$, meaning that no solitary waves form at exactly parallel or perpendicular propagation. Furthermore, the width increases when $\theta$ increases from zero, attains its maximum at some angle $\theta \approx 54.7^{\circ}$, and then decreases for further increases in $\theta$. Considering that the amplitude $\propto 1 / \cos \theta$, one sees that the KAW pulses propagating at small angles to the external magnetic filed tend to be weak and narrow ones.

\section{NONLINEAR SCHRODINGER EQUATION AND ROGUE WAVE SOLU- TIONS}

To study the modulational instability of the weakly nonlinear wave packets described by the KdV equation (22), we first make the dispersion coefficient positive by making a simple transform $\bar{\psi}=-\psi, \bar{\tau}=\tau$ and $\bar{\xi}=-\xi$, which will not change the solution in essence [53]. Actually, the KdV equation can be equally applied to a medium with negative dispersion (when the phase velocity of waves decreases with increasing wave number) as well as to a medium with positive dispersion, the difference being only in the direction in which the $\xi$ - axis is directed. With this transform the $\mathrm{KdV}$ equation retains its original form, but the resulting nonlinearity and dispersion coefficients become $\bar{A}=A$ and $\bar{B}=-B$. Next we consider solutions to the resulting $\mathrm{KdV}$ equation in the form of a weakly modulated sinusoidal wave $\bar{\psi}$ (for details of the technique, see e.g., [54, 55])

$$
\bar{\psi}=\sum_{n=1}^{\infty} \epsilon^{n} \sum_{l=-\infty}^{+\infty} \psi_{l}^{n}(\zeta, \chi) e^{\iota l(k \bar{\xi}-\omega \bar{\tau})}
$$

where $k$ is the carrier wave number and $\omega$ is the angular frequency for the given KAW. The stretched variables $\zeta$ and $\chi$ are chosen as

$$
\zeta=\epsilon\left(\bar{\xi}+v_{g} \bar{\tau}\right) \quad \text { and } \quad \chi=\epsilon^{2} \bar{\tau}
$$


where $v_{g}$ is the group velocity. By standard practice, we assume that fast scales enter into our discussion via the phase $(k \xi-\omega \tau)$ only, while slow scales $(\zeta, \chi)$ do so only in the form of the arguments of the $l$-th harmonic amplitude $\psi_{l}^{n}$. For $\psi(\zeta, \chi)$ to be real, one must require that $\psi_{-l}^{n}=\psi_{l}^{n *}$ where ${ }^{*}$ denotes complex conjugate. Substituting Eqs.(29) and (30) into Eq.(22), one readily finds

$$
\begin{aligned}
& -\iota l \omega \psi_{l}^{(n)}+v_{g} \frac{\partial \psi_{l}^{(n-1)}}{\partial \chi}+\frac{\partial \psi_{l}^{(n-2)}}{\partial \chi}+\bar{A} \sum_{n^{\prime}=1}^{\infty} \epsilon^{n} \sum_{l^{\prime}=-\infty}^{+\infty}\left(\iota k l \psi_{l}^{(n)} \psi_{l-l^{\prime}}^{\left(n-n^{\prime}\right)}+\psi_{l-l^{\prime}}^{\left(n-n^{\prime}\right)} \frac{\partial \psi_{l}^{(n)}}{\partial \zeta}\right) \\
& +\bar{B}\left(-\iota l^{3} k^{3} \psi_{l}^{(n)}-3 l^{2} k^{2} \frac{\partial \psi_{l}^{(n-1)}}{\partial \zeta}+3 \iota l k \frac{\psi_{l}^{(n-2)}}{\partial \zeta^{2}}\right)+\bar{B} \frac{\partial \psi_{l}^{(n-3)}}{\partial \zeta^{3}}=0 .
\end{aligned}
$$

With $(n, l)=(1,1)$ one finds that

$$
\omega=-\bar{B} k^{3}
$$

while the equations with $(n, l)=(2,1)$ give the group velocity as

$$
v_{g}=3 \bar{B} k^{2}
$$

Proceeding to $(n, l)=(2,2)$ and $(2,0)$, one finds that

$$
\psi_{2}^{(2)}=\frac{\bar{A}}{6 \bar{B} k^{2}} \psi_{1}^{(1)}, \quad \psi_{0}^{(2)}=-\frac{\bar{A}}{v_{g}}\left|\psi_{1}^{(1)}\right| .
$$

Finally, substituting the above derived expressions into the $(l=1)$ component of the third-order part of the reduced equations, one obtains the following nonlinear Schrödinger equation expressed in $\Psi \equiv \psi_{1}^{(1)}$,

$$
\iota \frac{\partial \Psi}{\partial \chi}+P \frac{\partial^{2} \Psi}{\partial \zeta^{2}}+Q|\Psi|^{2} \Psi=0,
$$

where

$$
P=6 \bar{B} k, \quad \text { and } \quad Q=\frac{\bar{A}^{2}}{6 \bar{B} k}
$$

are the dispersion and nonlinear coefficients, respectively. To study rogue waves, a rational solution to Eq.(35) reads (see e.g., [56])

$$
\Psi=\frac{1}{\sqrt{Q}}\left[\frac{4(1+2 \iota \chi)}{1+4 \chi^{2}+4 \zeta^{2} / P}\right] \exp \iota \chi,
$$

which predicts the concentration of KAW energy into a small region due to the nonlinear properties of the plasma medium. Rogue waves are usually an envelope of a carrier wave with a wavelength smaller than in the central region of the envelope. 
Based on the linear stability analysis [57, 58], it is observed that the waves as described by the nonlinear Schrodinger equation are modulationally unstable when $P / Q>0$ and when the modulation wave number satisfies $K^{2}<\left|\psi_{0}\right|^{2}(2 Q) / P$, where $\psi_{0}$ is the amplitude of the carrier waves. Furthermore, the maximum growth rate is given by $Q\left|\psi_{0}\right|^{2}$ and is attained at $K=\sqrt{\frac{Q}{P}}\left|\psi_{0}\right|$. Two types of stationary solutions are possible: (i) Unstable solutions called bright envelope solitons when $P / Q>0$ and (ii) Stable solutions called dark envelope solitons when $P / Q<0$. Evidently, the case examined in the present study falls in the first category.

To show how the wave envelope depends on the electron superthermality, Figure 4 shows the $\Psi$ profiles as a function of $\zeta$ and $\chi$ for (a) $\kappa=1.6$, (b) $\kappa=2$ and (c) $\kappa=4$ when $\beta=0.05, \theta=20^{\circ}$, and $k=0.9$. It is clear that both the amplitude and width of the envelope increase when $\kappa$ increases, meaning that the more pronounced the deviation from a Maxwellian, the weaker the rogue waves. Thus one can say that rogue waves for Maxwellian plasmas correspond to higher energy than for superthermal plasmas.

Figure 5 examines how the rogue waves depend on the plasma $\beta$ by presenting the $\Psi$ profiles as a function of $\zeta$ and $\chi$ for (a) $\beta=0.01$, (b) $\beta=0.05$ and (c) $\beta=0.1$ when $\kappa=2, \theta=20^{\circ}$ and $k=0.9$. One can readily see that rogue waves have a sensitive dependence on the plasma $\beta$ for the parameters chosen here, with the tendency being that increasing $\beta$ substantially increases the amplitude of the envelope. Hence one expects to see stronger and narrower envelops in the regions with higher $\beta$. Although not shown, the amplitude of rogue waves is found to increase with increasing obliqueness.

\section{CONCLUSIONS}

We have presented a theoretical study on the propagation dynamics of solitary kinetic Alfvén waves (KAWs) in low beta plasmas characterized by a superthermally distributed electron population, modeled by a kappa-type distribution. The standard reductive perturbation method was employed to derive the Korteweg de-Vries (KdV) equation. We have traced the effects of the electron superthermality, plasma $\beta$ and obliqueness on the characteristics of solitary KAWs.

An exact $\mathrm{KdV}$ equation has been derived for the first time using the reductive perturbation method for kinetic Alfvén solitary waves. Only compressive 
solitons are permitted. The Hasegawa-Mima [7] results are recovered when the electron distribution approaches a Maxwellian. The electron superthermality makes solitary pulses narrower and weaker, relative to a Maxwellian plasma. A magnetic field makes solitons wider but has no effect on the pulse amplitude. With increasing obliqueness relative to the external magnetic field, solitons become taller and wider. When the wave vector is exactly parallel to the external magnetic field, no soliton forms, for in this case the basic equations takes the form of ideal MHD, which is non-dispersive in nature for uniform plasmas. To study rogue wave solutions for KAWs, a nonlinear Schrödinger wave equation was derived from the KdV equation by using a standard perturbation method. It is observed that the electron superthermality lowers the rogue wave amplitude, meaning that kinetic Alfvén rogue waves can have higher energies in Maxwellian plasmas than in superthermal plasmas. Furthermore, it is observed that plasmas with higher $\beta$ support stronger rogue waves.

Our findings may help explain and interpret nonlinear oscillations in low $\beta$ plasmas such as planetary magnetospheres and the solar wind.

\section{Acknowledgments}

We are grateful to the anonymous referee whose comments helped improve this manuscript substantially. This research is supported by the 973 program 2012CB825601, the National Natural Science Foundation of China (40904047, 41174154, 41274176, and 41274178), the Ministry of Education of China (20110131110058 and NCET-11-0305), and by the Provincial Natural Science Foundation of Shandong via Grant JQ201212.

[1] A. Hasegawa and C. Uberoi, The Alfvén Wave (Technical Information Center, U. S. Department of Commerce, Springfield, Virginia, 1982).

[2] N. F. Cramer, The Physics of Alfvén Waves, Wiley-VCH, Berlin, 2001.

[3] J. W. Belcher and L. Davis, Jr., J. Geophys. Res. 76, 3534 (1971)

[4] R. Z. Sagdeev and A. A. Galeev, Nonlinear Plasma Theory (Benjamin, New York, 1969), p. 8. 
[5] P. Louarn, J. E. Wahlund, T. Chust, H de Feraudy, A. Roux, B. Holback, P. O. Dovner, A. I. Eriksson, and G. Holmgren, Geophys. Res. Lett. 21, 1847 (1994).

[6] P. O. Dovner and G. Holmgreen, Geophys. Res. Lett. 21, 1827 (1994).

[7] A. Hasegawa and K. Mima, Phys. Rev. Lett. 37, 690 (1976).

[8] M. Y. Yu and P. K. Shukla, Phys. Fluids 21, 1457 (1978).

[9] P. K. Shukla, H. U. Rahaman and R. P. Sharma, J. Plasma Phys. 28, 125 (1982).

[10] M. K. Kalita and B. C. Kalita, J. Plasma Phys. 35, 267 (1986).

[11] K. P. Das, L. P. J. Kamp and F. W. Sluijter, J. Plasma Phys. 41, 171 (1989).

[12] G. Rowlands, J. Plasma Phys. 3, 567 (1969)

[13] E. Infeld and G. Rowlands, J. Plasma Phys. 10, 293 (1973).

[14] G. Ghosh and K. P. Das, J. Plasma Phys. 51, 95 (1994).

[15] D. J. Wu, D. Y. Wang and C. G. Falthammar, Phys. Plasmas 2, 4476 (1995).

[16] D. J. Wu and D. Y. Wang, Phys. Plasmas 3, 4304 (1996).

[17] D. J. Wu, G. L. Huang and D. Y. Wang, Phys. Plasmas 3, 2879 (1996).

[18] X. Y. Wang, X. Y. Wang, Z. X. Liu and Z. Y. Li, Phys. Plasmas 5, 3477 (1998).

[19] X. Y. Wang, X. Y. Wang, Z. X. Liu and Z. Y. Li, Phys. Plasmas 5, 4395 (1998).

[20] D. J. Wu and J. K. Chao, Nonlin. Processes Geophys. 11, 631 (2004).

[21] L. Yang and D. J. Wu, Phys. Plasmas 12, 112901 (2005).

[22] M. A. Mahmood, A. M. Mirza, P. H. Sakanaka and G. Murtaza, Phys. Plasmas 9, 3794 (2002).

[23] S. Mahmood and H. Saleem, Phys. Plasmas 15, 114504 (2008).

[24] M. H. Woo, C.M. Ryu and C. R. Choi, Phys. Plasmas 17, 053707 (2010).

[25] S. P. Christon, D. G. Mitchell, D. J. Williams, L. A.Frank, C. Y. Huang, and T. E. Eastman, J. Geophys. Res. 93, 2562, (1988).

[26] M. Maksimovic, V. Pierrard, and P. Riley, Geophys. Res. Lett. 24, 1511, (1997).

[27] M. P. Leubner, Phys. Plasmas 11, 1308 (2004).

[28] A. Hasegawa, K. Mima, and M. Duong-van, Phys. Rev. Lett. 54, 2608 (1985).

[29] V. M. Vasyliunas, J. Geophys. Res. 73, 2839 (1968).

[30] M. A. Hellberg and R. L. Mace, Phys. Plasmas 9, 1495 (2002).

[31] T. K. Baluku and M. A. Hellberg, Phys. Plasmas 15, 123705 (2008).

[32] N. S. Saini, I. Kourakis and M. A. Hellberg, Phys. Plasmas 16, 062903 (2009).

[33] T. S. Gill, A. S. Bains and C. Bedi, Phys. Plasmas 17, 013701 (2010). 
[34] T. S. Gill, A. S. Bains, N. S. Saini and C. Bedi, Phys. Letts. A 374, 3210 (2010).

[35] A. Bandyopadhyay and K. P. Das, Phys. Plasmas 7, 3227 (2000).

[36] R. Roychoudhury, J. Plasma Phys. 67, 199 (2002).

[37] R. Gogoi and M. Khan, Phys. Plasmas 17, 112311 (2010).

[38] Y. Liu, S. Q. Liu and B. Dai, Phys. Plasmas 18, 092309 (2011).

[39] B. Kibler, J. Fatome, C. Finot, G. Millot, F. Dias, G. Genty, N. Akhmediev, and J. M. Dudley, Nat. Phys. 6, 790 (2010).

[40] D. R. Solli, C. Ropers, P. Koonath, and B. Jalali, Nature 450, 1054 (2007).

[41] R. Hohmann, U. Kuhl, H.-J. Stockmann, L. Kaplan, and E. J. Heller, Phys.Rev. Lett. 104, 093901 (2010).

[42] L. Stenflo and M. Marklund, J. Plasma Phys. 76, 293295 (2010).

[43] W. M. Moslem, P. K. Shukla, and B. Eliasson, EPL 96, 25002 (2011).

[44] U. M. Abdelsalam, W. M. Moslam, A. H. Khater and P. K. Shukla, Phys. Plasmas 18, 092305 (2011).

[45] E. I. El-Awady and W. M. Moslem, Phys. Plasmas 18, 082306 (2011).

[46] R. Sabry, W. M. Moslem and P. K. Shukla, Phys. Plasmas 19, 122903 (2012).

[47] P. K. Shukla and W. M. Moslem, Phys. Letts. A 376, 1125 (2012).

[48] A. Panwar, H. Rizvi and C. M. Ryu, Phys. Plasmas 20, 082101 (2013).

[49] E. I. El-Awady, H. Rizvi, W. M. Moslem, S. K. El-Labany, A. Raouf and M. Djebli, Astrophys. Space Sci. 349, 5 (2014).

[50] B. B. Kadomtsev, Plasma Turbulence (Academic, New York, 1965), p.82.

[51] M. N. Kadijani, H. Abbasi and H. H. Pajouh, Plasma Phys. Control. Fusion 53, 025004 (2011).

[52] S. Sultana, I. Kourakis, N. S. Saini and M. Hellberg, Phys. Plasmas 17, 032310 (2010).

[53] V. Yu. Belashov and S. V. Vladimirov, Solitary waves in Dispersive Complex Media (SpringerVerlag Berlin Heidelberg 2005) p. 22.

[54] K. Shimizu and Y. H. Ichikawa, J. Phys. Soc. Jpn 33, 789 (1972).

[55] S. K. El-Labany, J. Plasma Phys. 54, 295 (1995).

[56] W. M. Moslam, Phys. Plasmas 18, 032301 (2011).

[57] M. R. Amin, G. E. Morfill, and P. K. Shukla, Phys. Rev. E 58, 6517(1998).

[58] A. S. Bains, M. Tribeche, and T. S. Gill, Phys. Lett. A 375, 20592063 (2011). 


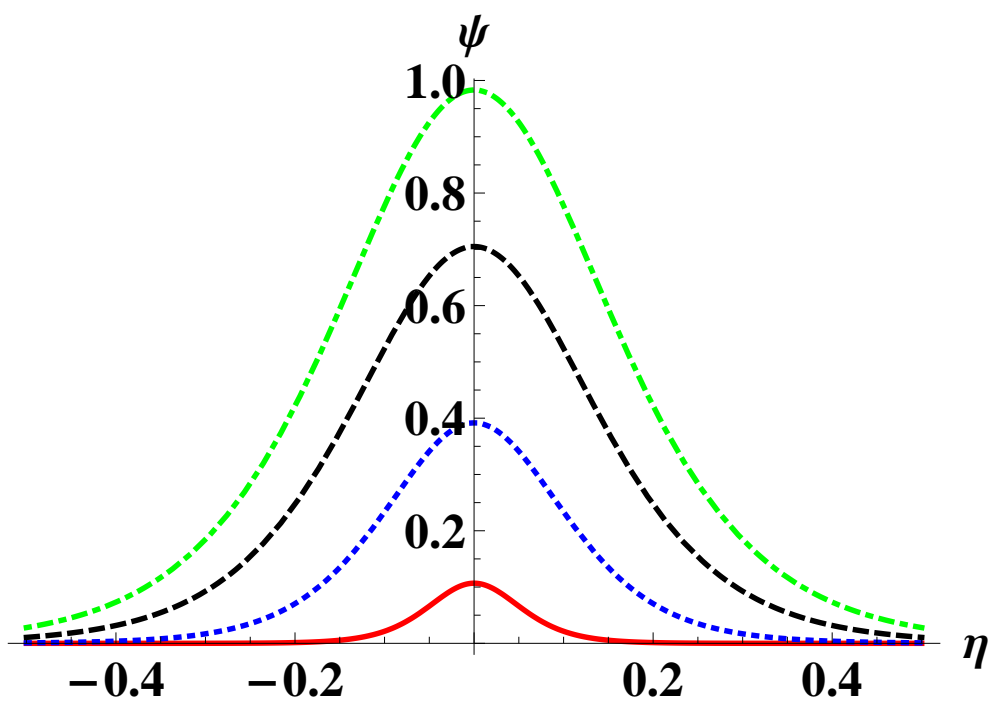

FIG. 1: (Color online) Profiles of solitary kinetic Alfvén waves for a range of $\kappa$ values characterizing the electron superthermality at fixed values of plasma $\beta=0.05$ and obliqueness $\theta=40^{\circ}$. The solid curve corresponds to $\kappa=1.6$, dotted curve to $\kappa=2$, dashed curve to $\kappa=3$, and dot-dashed curve to $\kappa=10$. 


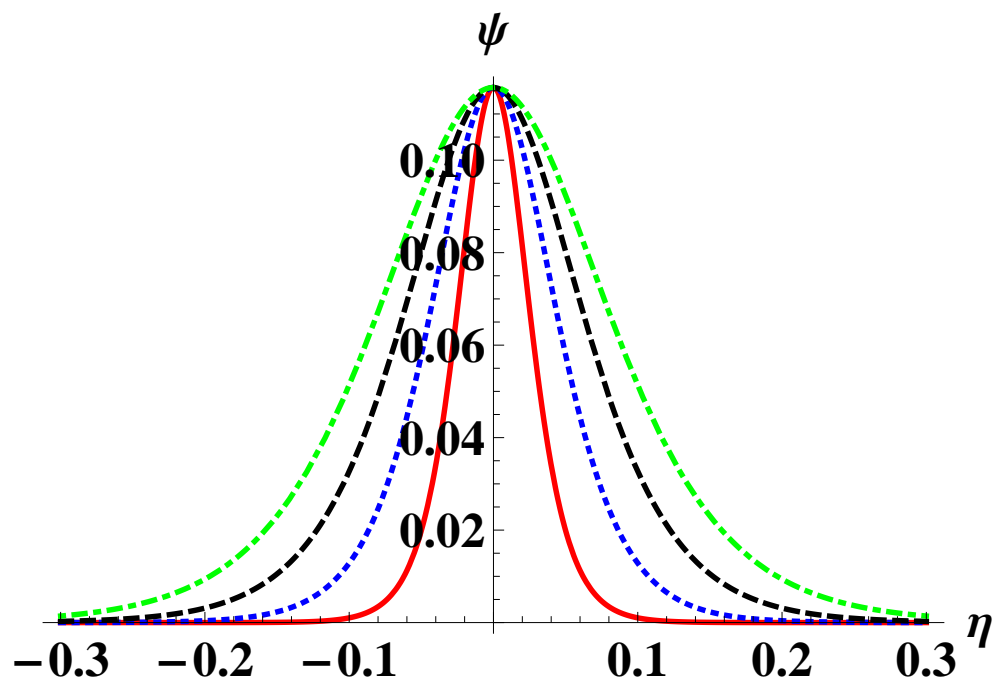

FIG. 2: (Color online) Profiles of solitary kinetic Alfvén waves for a range of values of the plasma $\beta$ at fixed values of electron superthermality $\kappa=2$ and obliqueness $\theta=45^{\circ}$. The solid curve corresponds to $\beta=0.01$, dotted curve to $\beta=0.03$, dashed curve to $\beta=0.06$, and dot-dashed curve to $\beta=0.1$. 


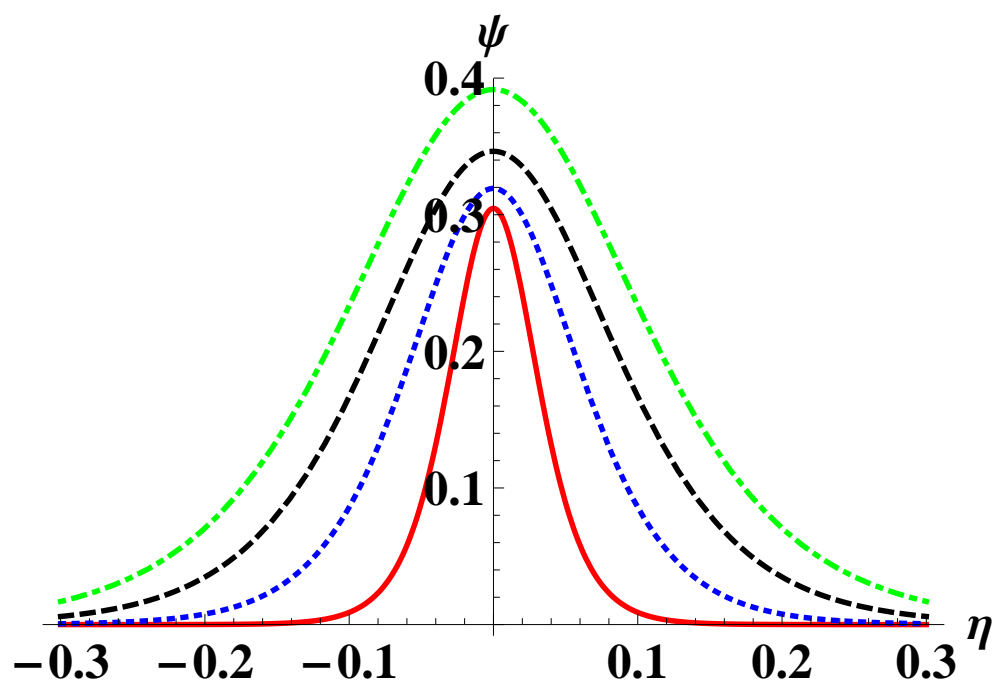

FIG. 3: (Color online) Profiles of solitary kinetic Alfvén waves for a range of values of obliqueness $\theta$ at fixed values of electron superthermality $\kappa=2$ and plasma $\beta=0.05$. The solid curve corresponds to $\theta=10^{\circ}$, dotted curve to $\theta=20^{\circ}$, dashed curve to $\theta=30^{\circ}$, and dot-dashed curve to $\theta=40^{\circ}$. 
(a)

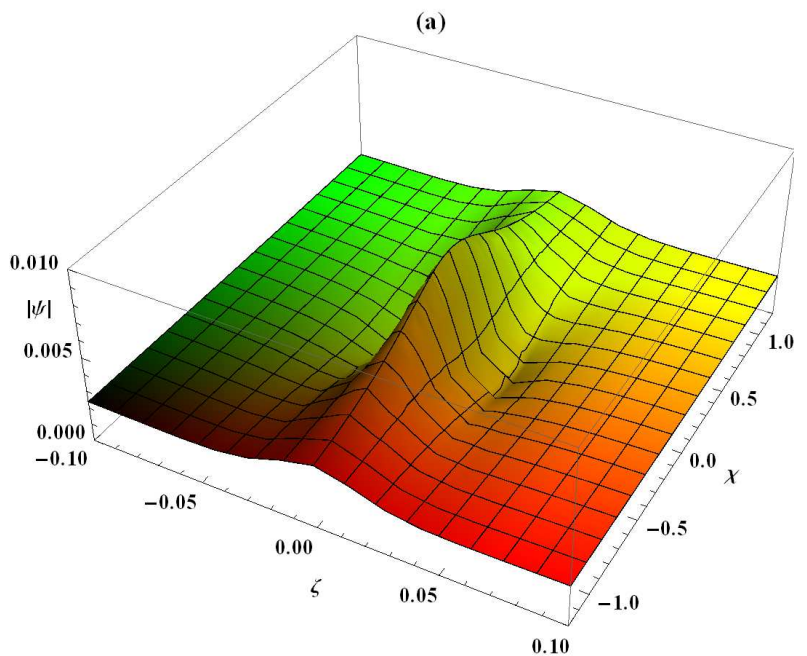

(c)

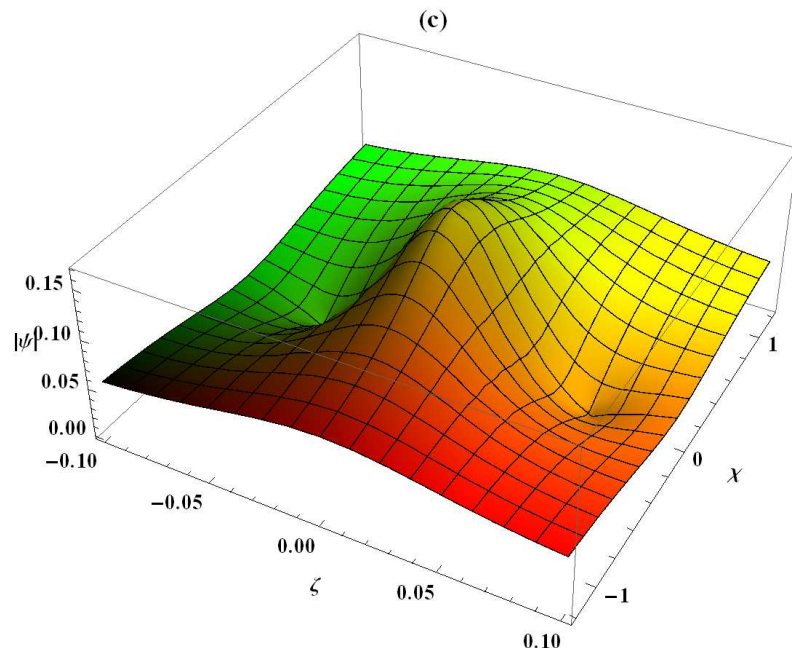

(b)

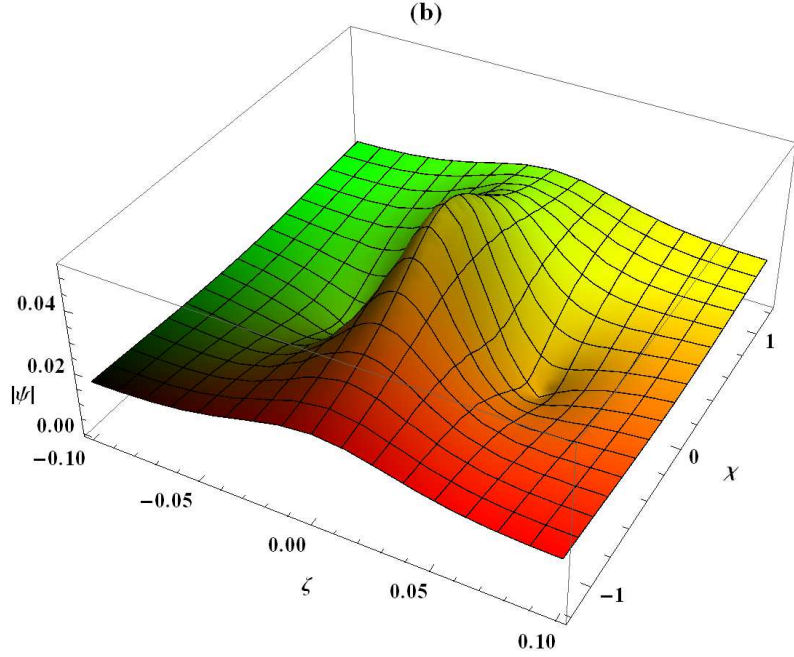

FIG. 4: (Color online) The rogue wave profile $|\Psi|$ with respect to $\chi$ and $\zeta$ for different values of $\kappa$ : (a) $\kappa=1.6$, (b) $\kappa=2$ and (c) $\kappa=4$. Here $\beta=0.05, \theta=20$ and $k=0.9$. 
(a)

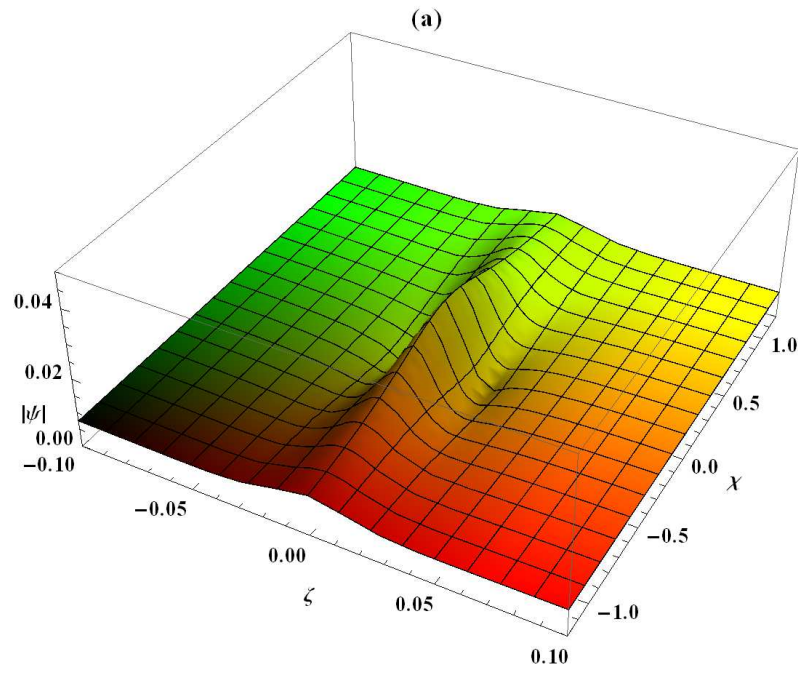

(c)

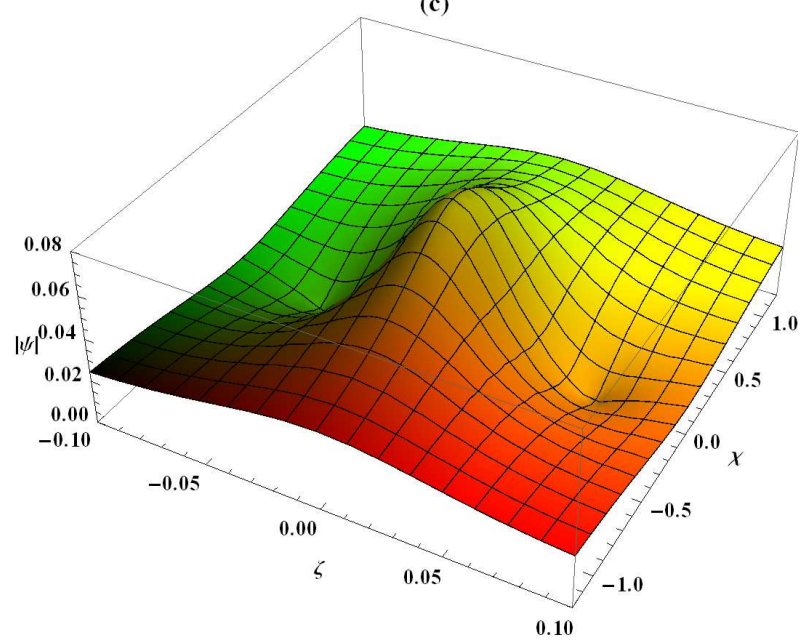

(b)

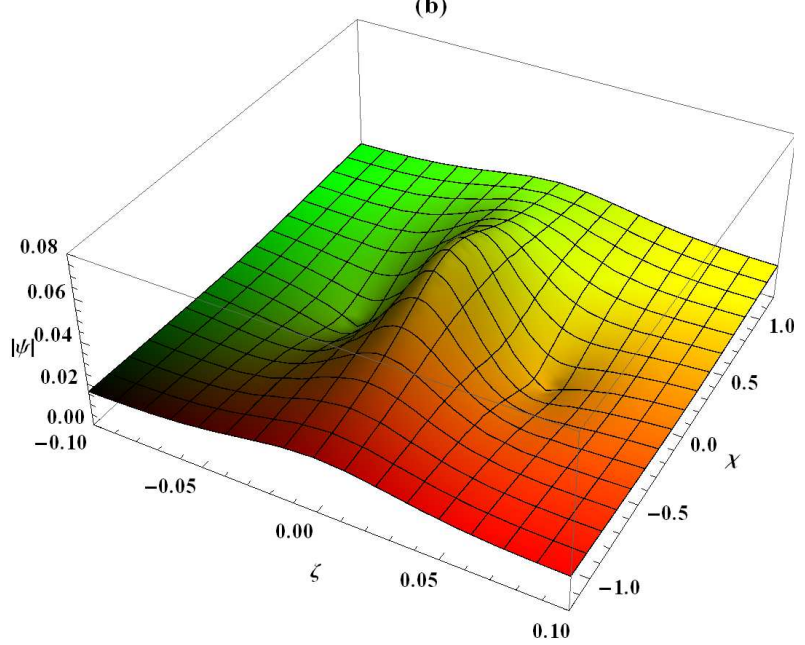

0.10

FIG. 5: (Color online) The rogue wave profile $|\Psi|$ with respect to $\chi$ and $\zeta$ for different values of $\beta$ :

(a) $\beta=0.01$, (b) $\beta=0.05$ and (c) $\beta=0.1$. Here $\kappa=2, \theta=20^{\circ}$ and $k=0.9$. 